\title{
A time domain spherical near-field measurement facility for UWB antennas employing a hardware gating technique
}

\author{
M. D. Blech ${ }^{1}$, M. M. Leibfritz ${ }^{2}$, R. Hellinger ${ }^{3}$, D. Geier ${ }^{1}$, F. A. Maier ${ }^{4}$, A. M. Pietsch ${ }^{1}$, and T. F. Eibert \\ ${ }^{1}$ Institut für Hochfrequenztechnik, Universität Stuttgart, Stuttgart, Germany \\ ${ }^{2}$ Rohde \& Schwarz GmbH \& Co. KG, Munich, Germany \\ ${ }^{3}$ Robert Bosch GmbH, Leonberg, Germany \\ ${ }^{4}$ Hüttinger Elektronik, Freiburg, Germany \\ ${ }^{5}$ Lehrstuhl für Hochfrequenztechnik, Technische Universität München, Munich, Germany
}

\begin{abstract}
A spherical near-field antenna measurement facility employing a time domain hardware gating technique is presented. On-off keyed sinusoidal impulses are used as stimuli requiring wideband antennas with a bandwidth in excess of $400 \mathrm{MHz}$. The received signal is evaluated in the time interval after reaching the steady state and before multipath components arising in the non-ideal anechoic chamber distort the signal.

An application specific pulse generator synthesizing sinusoidal impulses with a sub-nanosecond settling time and a low-cost equivalent time (ET) sampling receiver developed and optimized for this particular purpose are described.

Measurement results of typical ultra-wideband (UWB) antennas show a significant improvement of the measured antenna pattern compared to conventional techniques.
\end{abstract}

\section{Introduction}

The far-field pattern of antennas is described by the characteristic of the amplitude of the E- or H-field (ANSI, 1979). This characteristic can be measured in a test cite requiring large dimensions to obtain far-field conditions. Very often the requirements can only be met by outdoor measurements, which can be affected by the wheater as well as electromagnetic interferences. One solution to overcome these drawbacks are near-field measurements with a subsequent nearfield to far-field transformation (Hansen, 1988). They can be carried out in comparably small indoor test sites equipped

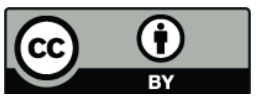

Correspondence to: M. D. Blech (mail@ihf.uni-stuttgart.de) with absorbing material. However, the absorbers still cause some reflections resulting in multipath propagation in the anechoic chamber that distorts the received signal. In the employed near-field to far-field transformation measurement errors in the amplitude and the phase of the field radiated by the antenna under test (AUT) also propagate to the far-field pattern.

Several techniques have been developed to cancel out the multipath components in the received signal. Hardware (HW) gating employing a fast impulse or step generator and a sampling oscilloscope (Alenkowicz and Levitas, 2000) is one typical approach to measure the received signal by an oscilloscope and to gate out the undesired signal components (DeJough et al., 1997; Levitas and Ponomarev, 1996; Klembowski et al., 1996; Levitas, 2006). However, this technique requires antennas exhibiting a bandwidth in excess of $10 \mathrm{GHz}$ to work properly.

In conventional software (SW) gating procedures (Henderson et al., 1989; Lestari et al., 2005) the received signal is sampled in the frequency domain using a vector network analyzer (VNA). In a post-processing step the inverse discrete Fourier transform (DFT) is applied and the resulting time domain signal is gated. After canceling the multipath components of the signal the data can be used for the determination of the antenna pattern at the desired frequency. This technique produces non-physical results if the delay spread in the measurement environment is too large for the chosen frequency spacing of the acquired samples.

Novel SW gating principles evaluating a frequency domain signal (Fourestie et al., 1999; Loredo et al., 2004) as well as high-resolution techniques using the shorttime Fourier transform (Fourestie and Altman, 2001) have been developed. These techniques can be applied to antennas

Published by Copernicus Publications on behalf of the URSI Landesausschuss in der Bundesrepublik Deutschland e.V. 


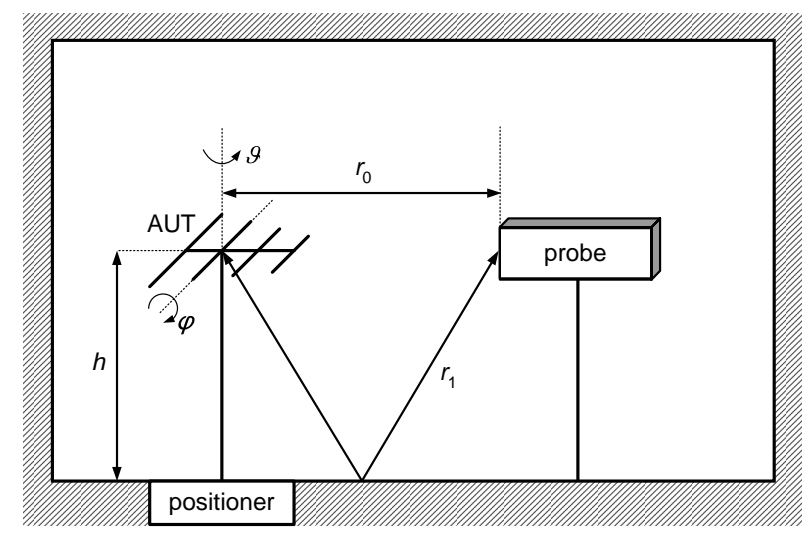

Fig. 1. Multipath propagation in the anechoic chamber.

with less bandwidth than required by the conventional SW gating approach. However, acquiring a frequency domain signal over a wide bandwidth with a high resolution causes a long measurement period, even using state of the art instrumentation. Another technique based on the design of a truncated finite impulse response (FIR) filter in the z-domain representing the channel between the AUT (Dadic and Zentner, 2007) has been introduced. This technique allows nonuniform frequency spacing and assures causal impulse responses.

In Hartmann and Fasold (1998) a HW gating procedure employing ultra fast PIN-switches to gate a continuous wave (CW) signal is presented yielding an enhancement of measurements conducted in compensated compact ranges. Due to the comparably slow switching time of approximately $1 \mathrm{~ns}$ of ultra-fast PIN-switches this technique can only be applied in large test sites, where the measurement signal must travel large distances.

In order to use this technique for spherical near-field measurements it is necessary to synthesize a sinusoidal impulse with a fast settling time (Leibfritz et al., 2007). The pulse generator presented in Leibfritz et al. (2007) has now been redesigned to increase the operating frequency to the band from 1.5 to $8 \mathrm{GHz}$. Its output signal achieves a settling time of $150 \mathrm{ps}$. For the reduction of the cost and the acceleration of the measurement speed a new application specific ET sampling receiver has been developed. 3-dimensional measurement results show a significant enhancement of the acquired antenna patters in amplitude and phase.

An overview of the developed HW gating technique is presented in Sect. 2. The time domain antenna measurement system, focusing on the equivalent time (ET) sampling receiver, is described in Sect. 3. In Sect. 4 some exemplary measurement results are presented before the paper is concluded in Sect. 5.

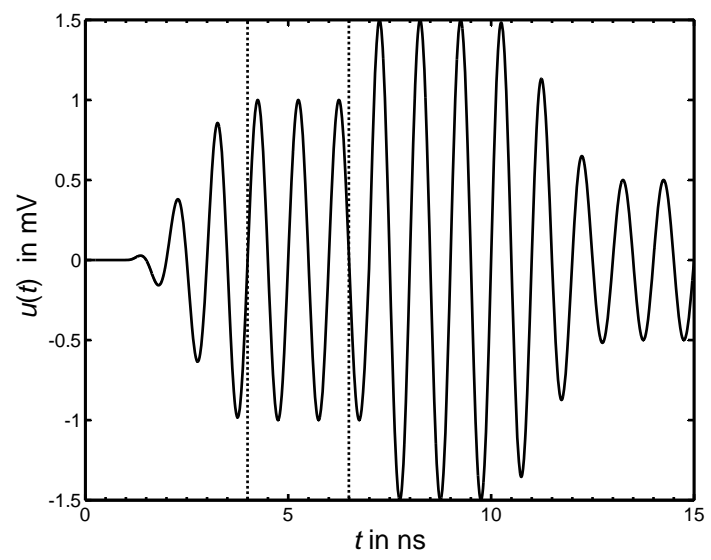

Fig. 2. Time interval $t_{\text {meas }}$ (enclosed by dashed lines) in which the pulsed sinusoidal signal is evaluated.

\section{Hardware gating technique}

The hardware gating technique employed makes use of the time difference of arrival (TDOA) of the line of sight (LOS) signal component and the non line of sight (NLOS) components which arise due to multipath propagation in the nonideal measurement environment. This scenario is shown in Fig. 1. The AUT is depicted on the left hand side. It is attached to a spherical positioner which orientates the antenna in both $\vartheta$ and $\varphi$. The AUT is mounted at the height $h$ and at the distance $r_{0}$ from the probe. The distance $r_{0}$ is the path that the LOS component travels from the AUT to the probe. The first NLOS component which disturbs the received signal is a first order reflection from the ground floor. This ray has to travel the distance $r_{1}$. The TDOA

$\Delta t=\frac{r_{1}}{c_{0}}-\frac{r_{0}}{c_{0}}=\frac{2 \sqrt{\left(\frac{r_{0}}{2}\right)^{2}+h^{2}}}{c_{0}}-\frac{r_{0}}{c_{0}}$

between the reception of the LOS and the NLOS signal results in $5.66 \mathrm{~ns}$ assuming an antenna height $h=1.5 \mathrm{~m}$ and a distance $r_{0}=1.8 \mathrm{~m}$. The speed of light is denoted by $c_{0}$. As the used antennas are band limited they affect the rise time of the envelope of the received signal. As a good approximation the rise time $t_{\mathrm{r}}$ can be assumed to be inversely proportional to the bandwidth $B$ of a bandpass (BP) of low order. In order to ensure a time interval $t_{\text {meas }} \geq 2 \mathrm{~ns}$ for the evaluation of the received signal, the antennas must have reached their steady state after the rise-time

$t_{r}=\Delta t-t_{\text {meas }}$,

which in this case is $3.66 \mathrm{~ns}$. Thus each of them must exhibit a $3 \mathrm{~dB}$ bandwidth

$B=\frac{\sqrt{2}}{t_{\mathrm{r}}}$ 
of at least $0.4 \mathrm{GHz}$, which results from the bandpass system consisting of two cascaded antennas with the same bandwidth. Due to this fact the HW gating technique discussed above is only capable of measuring antennas providing a significant bandwidth, where the requirements of the AUT with respect to their relative bandwidth decrease with increasing measurement frequency. A plot of a typical received voltage is given in Fig. 2. The dashed lines show the time interval $t_{\text {meas }}$ in which the signal is evaluated. It is precisely detected by means of correlation and evaluation of the signal's envelope, which is very robust even for low signal to noise ratios (SNR) employing the $\mathrm{CW}$ based technique.

\section{Antenna measurement system}

A brief overview of the near-field antenna test facility is given in Fig. 3. All application specific components denoted as blocks with double solid lines have been developed at the authors' institute to optimize the overall system performance.

A pulse generator synthesizes the required sinusoidal stimuli. The received signal can be acquired by either a commercially available real-time oscilloscope DSO81004A from Agilent Technologies or an application specific ET sampling receiver. A PC with a comfortable web interface controls the measurement system. The azimuth over elevation positioner which is driven by the motion control machine (MCM) software is supplied by Orbit.

\subsection{Pulse generator}

The redesigned pulse generator is an upgrade of the one described in Leibfritz et al. (2007). It is based on digital emitter coupled logic (ECL) circuits of the GigaComm series from ONSemiconductor used to switch a rectangular signal with the operating frequency, which can be up to $8 \mathrm{GHz}$. Generating a digital signal requires lowpass (LP) or BP filters with a constant group delay (Belyaev et al., 2000; Singh et al., 2007) to keep the waveform distortions low and to suppress the undesired harmonics. The synchronization and the adjustment of measurement parameters can be achieved in a comfortable way using a digital circuit. In the new pulse generator design the pulse width can be set to any value between 1 and $10 \mathrm{~ns}$ in steps of $10 \mathrm{ps}$, the pulse repetition frequency ranges from $40 \mathrm{MHz}$ down to $39 \mathrm{kHz}$, and the number of impulses can be any 32 bit integer value. The pulse generator can be controlled over a serial interface and provides the trigger signal for the sampling receiver.

\subsection{Equivalent time (ET) sampling receiver}

The antenna measurements can be performed using either a commercial real-time digitizing oscilloscope DSO81004A or an application-specific custom ET sampling receiver (Kahrs, 2003). This oscilloscope is the only one available to the authors exhibiting a bandwidth which is large enough. A

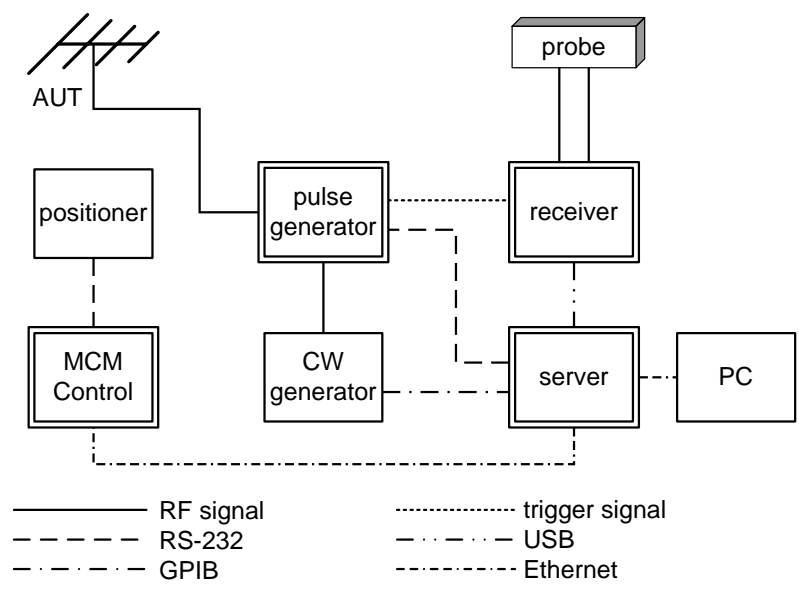

Fig. 3. Overview of the entire measurement system.

real-time oscilloscope is obviously not the best choice for this kind of application, anyway it delivers reasonable results (see Sect. 4). Gaining better specifications an application specific ET sampling receiver for the frequency range 1.5$9 \mathrm{GHz}$ has been developed which is optimized for resolution ( 8.0 bit), dynamic range (76.6 dB), and SNR (49.6 dB). Measurement speed is a key parameter in spherical antenna measurements, where an entire grid can consist of 7200 points $\left(\Delta \vartheta=3^{\circ}, \Delta \varphi=3^{\circ}\right)$ or more. Thus the acquisition of the field on an entire sphere can last longer than $24 \mathrm{~h}$. The main bottleneck besides positioning the AUT is the cycle duration for autoscaling, acquiring one waveform, and transferring it to the measurement server. Compared to the oscilloscope, the time consumption of that procedure is bisected by the ET receiver. The total costs of the application specific receiver sum up to approximately $5 \%$ of a general purpose instrument.

In Fig. 4 a block diagram of the ET receiver is depicted. The RF frontend consists of a commercially available electronic RF switch (Hittite Microwave Corp., 2007a) for the alternating use of both ports of a dual-polarized probe. It is followed by a wideband amplifier (Hittite Microwave Corp., 2007b) exhibiting a low noise figure and a constant group delay. Both aforementioned components are specified with a bandwidth of $20 \mathrm{GHz}$. The essential component of the receiver is a track \& hold (T\&H) amplifier (Inphi Corp., 2006) with a bandwidth of $13 \mathrm{GHz}$ that samples and holds the received signal.

In order to ensure a high dynamic range, a digitally controlled variable gain amplifier (VGA) is placed in between the $\mathrm{T} \& \mathrm{H}$ amplifier and a 16 bit pipelined analog to digital converter (ADC) (Analog Devices Inc., 2006), which is interfaced to a field programmable gate array (FPGA). This FPGA controls the RF switch, the VGA, and the time base unit. The latter is used for signal conditioning of the trigger impulse provided by the pulse generator, which is directly fed to the T\&H amplifier. The FPGA is connected to a PC via a bidirectional USB to serial converter, in order to control 


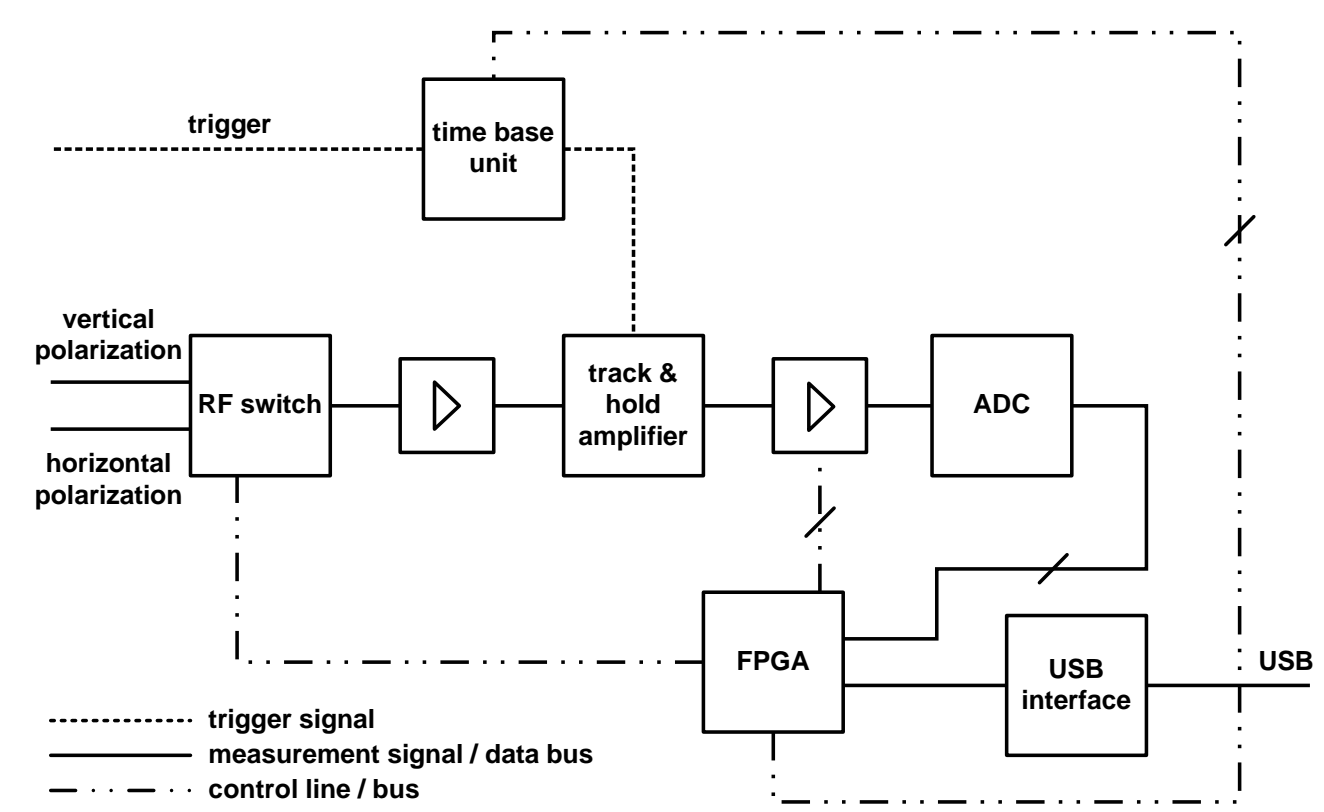

Fig. 4. Block diagram of the ET sampling receiver.

the receiver and to transfer measurement data rapidly to a host PC.

Noise and jitter are critical parameters in high speed sampling devices. Additive white Gaussian noise can easily be removed by averaging the measurement signal at each sample point. The resulting

$\mathrm{SNR}_{\mathrm{avg}}=\mathrm{SNR} \sqrt{n}$

is the SNR of one single measurement improved by the square root of the number of averaged samples $n$ (Paulter and Larson, 2005). In the presented receiver the number of averages can be set to any 16 bit integer value.

As long as the input bandwidth of the signal to be measured fulfills the condition

$t_{j} \leq \frac{1}{10 B}$,

where $B$ denotes the input bandwidth of the receiver and $t_{j}$ is the root mean squared (RMS) jitter of the trigger signal, the trigger induced jitter is negligible (Paulter and Larson, 2005). This holds for the presented receiver, where the input bandwidth

$B=\frac{1}{\sqrt{\frac{1}{B_{\text {Switch }}^{2}}+\frac{1}{B_{\text {Amplifier }}^{2}}+\frac{1}{B_{\text {T\&H }}^{2}}}}$

of the entire RF frontend depending on the bandwidths $B_{i}$ $(i=1,2, \ldots)$ of the single components is computed to be $9.57 \mathrm{GHz}$ and the jitter caused by the pulse generator, the time base unit, and the T\&H amplifier can be determined to be $2.2 \mathrm{ps}$. If condition (5) is not fulfilled, jitter causes a signal dependent bias in the acquired waveform (Souders et al., 1990). In this case, techniques considering both, noise and jitter, must be applied, as e.g. the Median Method (Paulter and Larson, 2005), spline based interpolating algorithms (Cox et al., 1993), or an approach of deconvolving time jitter from the measured waveform (Gans, 1983; Verspecht, 1994).

The time base unit used in the presented receiver is depicted in detail in Fig. 5. It consists of standard ECL devices that provide high speed signaling and low jitter. The applied repetitive trigger signal from the pulse generator is used for synchronization purposes by the FPGA. It is also used to generate a rectangular impulse combining the original step signal with a delayed copy by an XOR gate. The resulting impulse is fed through two cascaded digitally programmable variable delay lines (VDLs) (ON Semiconductor, 2006). These VDLs provide an adjustable delay of $0-20 \mathrm{~ns}$ in steps of $10 \mathrm{ps}$. The delayed trigger impulses initiate the T\&H amplifier to sample the received waveform.

However, the delay steps of the VDLs are temperature dependent and not ideally uniform. Therefore the VDLs are temperature controlled. The nonlinearity of the sampling grid is calibrated, whereas the nonuniform samples are interpolated to an equidistant grid employing a linear Lagrangian interpolation (Autar, 2003). As long as the nonuniform sample points deviate less than $T / 4$ from the desired uniform sampling interval $T$, the waveform can be approximated by a Lagrange interpolation (Higgins, 1976).

In order to ensure a proper frequency response, the RF frontend must be calibrated. Fig. 6 shows a comparison of the acquired pulsed sinusoidal waveforms of the DSO81004A and the ET receiver, respectively. Both are directly connected to 


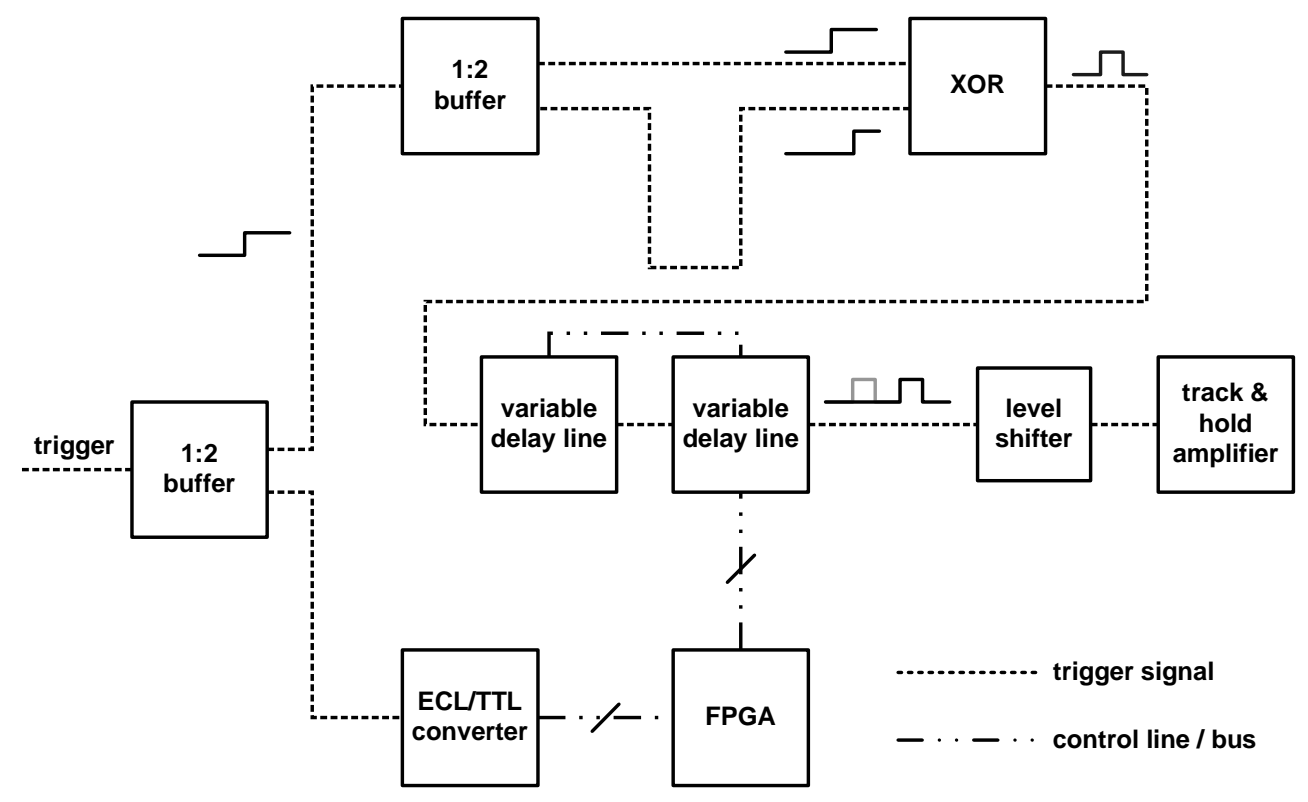

Fig. 5. Block diagram of the time base unit.

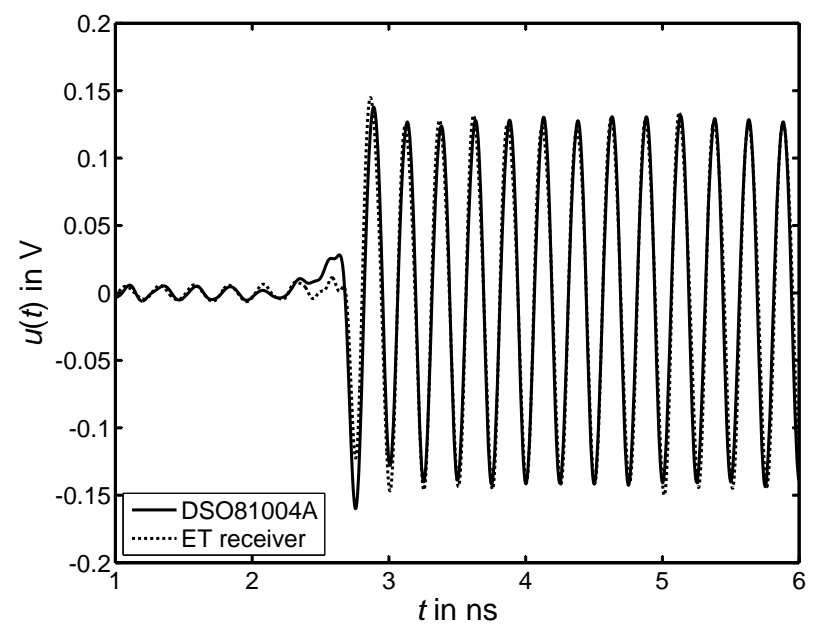

Fig. 6. Comparison of the measured test signal $(f=4 \mathrm{GHz})$ acquired by the real-time digitizing oscilloscope and the ET sampling receiver.

the pulse generator. As depicted, there is very little deviation between both measurement devices.

\section{Measurement results}

For the evaluation of the presented HW gating technique spherical near-field measurements have been carried out in the non-certified anechoic chamber of the institute employing the pulse generator described in Sect. 3.1, the DSO81004A oscilloscope, and an azimuth over elevation positioner from Orbit.
Two omnidirectional antennas, a discone and an UWB monopole antenna as well as two directional antennas, namely a Yagi and a Vivaldi antenna are employed to evaluate the influence of the measurement system on these types of antennas. All antennas are measured at a frequency of $1800 \mathrm{MHz}$, which is close to the lower limit of the measurement system of $1500 \mathrm{MHz}$. Measurement results for higher frequencies are expected to produce even better results than this worst case scenario as multiple periods of the sinusoidal signal can be evaluated in the limited time interval $t_{\text {meas }}$ which is gated.

The 3-dimensional far-field pattern of the antennas are simulated using CST Microwave Studio and FEKO, respectively. In order to obtain the far-field pattern from the three different measurement techniques investigated, namely an ordinary $\mathrm{CW}$ near-field measurement, a conventional SW gating technique, and the presented HW gating, the measurement data has to be post-processed in a subsequent near-field to far-field transformation. For an evaluation of the results, the patterns of all aforementioned techniques are compared to the simulation, which is taken as reference, in the range $\vartheta=90^{\circ}-160^{\circ}$. This part of the sphere is suitable for a comparison of the results as it does not contain the range $\vartheta=0^{\circ}-90^{\circ}$ and $\varphi=0^{\circ}-360^{\circ}$ which is affected by a system inherent blocking of the azimuth positioner which moves in between the AUT and the probe in this angular range. However, none of the described gating techniques can eliminate this effect. The angular range $\vartheta=160^{\circ}-180^{\circ}$ is also neglected as one of the nulls of the patterns is located here and the depth of the nulls of simulated patterns usually can not be achieved by antennas in practice. Thus taking into account this region of the null would falsify the entire comparison 


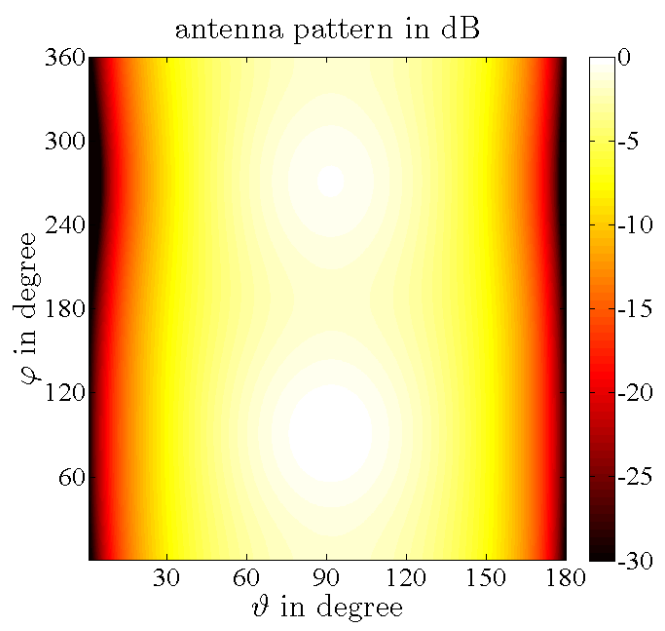

(a)

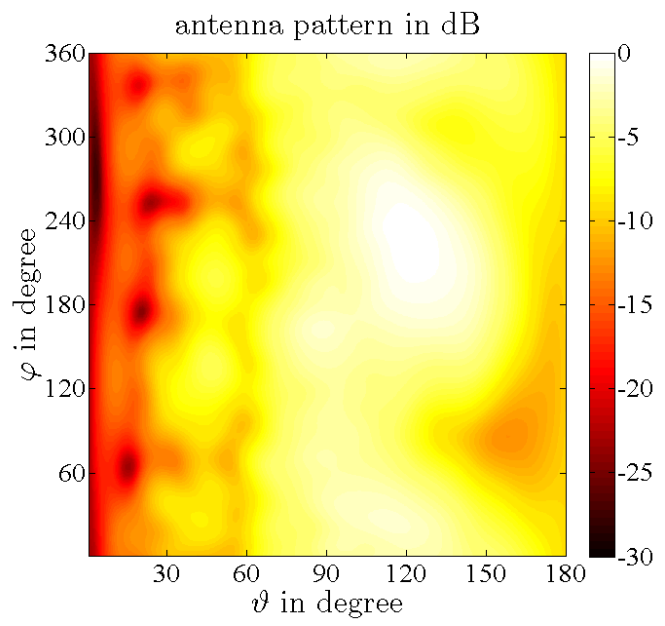

(c)

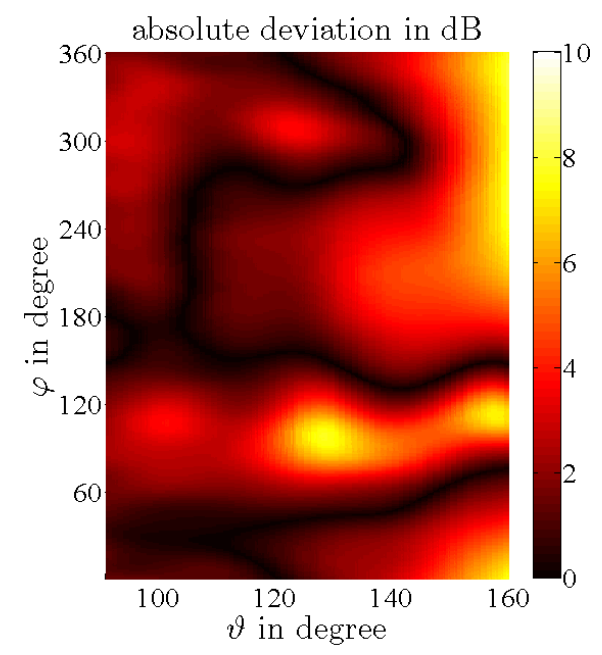

(e)

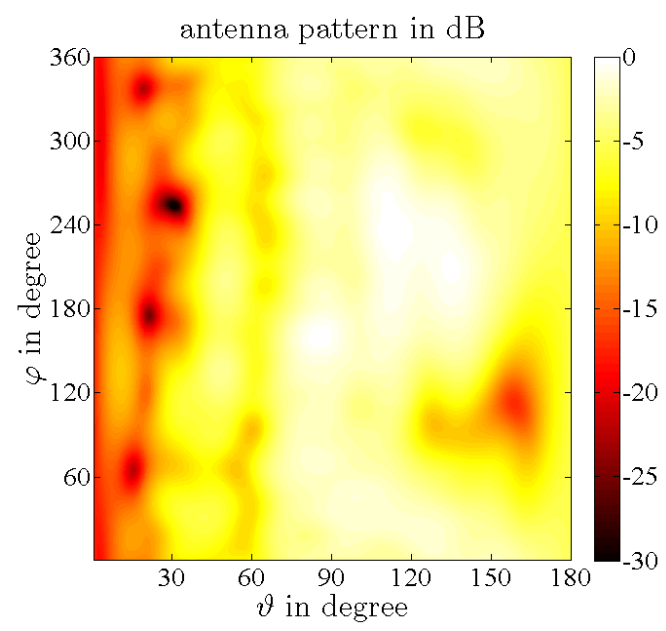

(b)

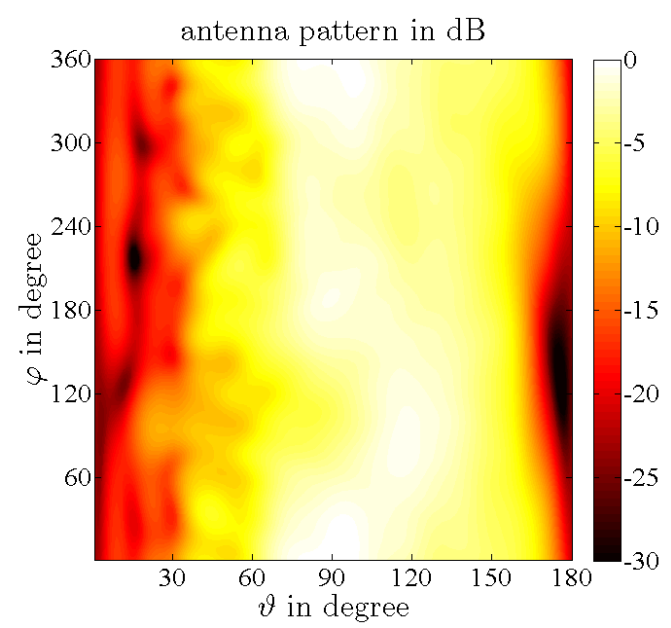

(d)

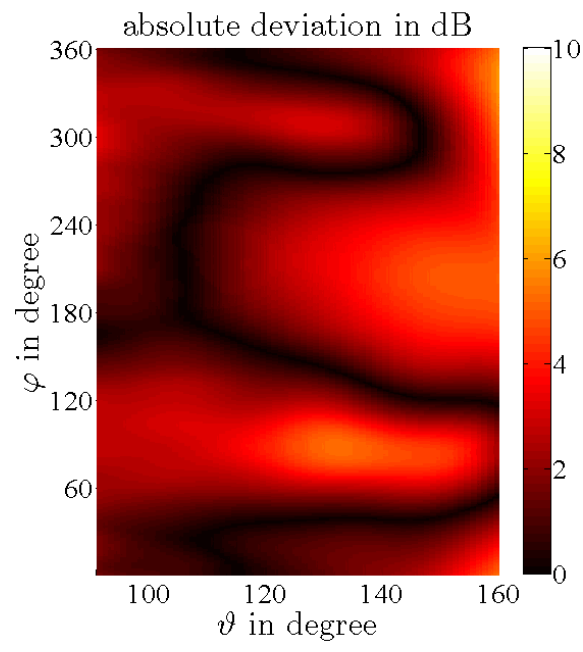

(f)

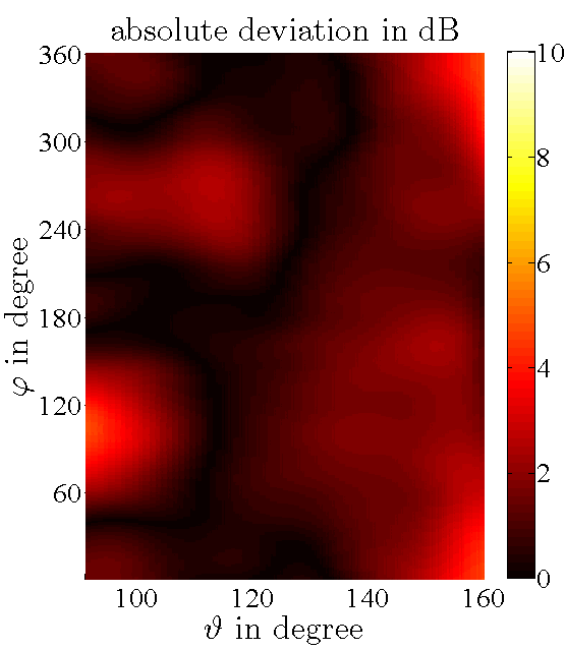

(g)

Fig. 7. The far-field pattern of a bulbous planar UWB antenna (simulation (a), standard near-field measurement (b), near-field measurement with SW gating (c), near-field measurement with HW gating (d)) and the deviation of the measurements from the simulation (standard near-field measurement (e), near-field measurement with SW gating (f), and near-field measurement with HW gating (g)). 
Table 1. Comparison of the absolute average deviation of the amplitude and the phase of the measurements from the simulated pattern taken as reference.

\begin{tabular}{cccc}
\hline & $\begin{array}{c}\text { conventional } \\
\text { frequency } \\
\text { domain } \\
\text { measure- } \\
\text { ment }\end{array}$ & $\begin{array}{c}\text { SW gating } \\
\text { measure- } \\
\text { ment }\end{array}$ & $\begin{array}{c}\text { HW gating } \\
\text { measure- } \\
\text { ment }\end{array}$ \\
\hline UWB & $2.38 \mathrm{~dB}$ & $2.07 \mathrm{~dB}$ & $1.22 \mathrm{~dB}$ \\
monopole & $53.5^{\circ}$ & $21.8^{\circ}$ & $9.9^{\circ}$ \\
antenna & $1.49 \mathrm{~dB}$ & $1.27 \mathrm{~dB}$ & $1.04 \mathrm{~dB}$ \\
discone & $63.1^{\circ}$ & $16.8^{\circ}$ & $12.0^{\circ}$ \\
antenna & $1.16 \mathrm{~dB}$ & $1.09 \mathrm{~dB}$ & $0.98 \mathrm{~dB}$ \\
Vivaldi & $89.2^{\circ}$ & $48.7^{\circ}$ & $75.7^{\circ}$ \\
antenna & $1.39 \mathrm{~dB}$ & $1.56 \mathrm{~dB}$ & $0.87 \mathrm{~dB}$ \\
Yagi antenna & $32.6^{\circ}$ & $43.1^{\circ}$ & $25.0^{\circ}$ \\
\hline
\end{tabular}

because of imperfectness of the antenna prototype in this small angular range.

The phase of the measured patterns is a good indicator to evaluate the influence of the different measurement techniques on the acquired data. Therefore the average absolute deviation of the measured phase from the simulation in the relevant angular range of $\vartheta=90^{\circ}-160^{\circ}$ is evaluated.

Exemplarily the simulated and measured patterns of the UWB monopole antenna (Schantz et al., 2005) are shown in this paper. The simulated radiation characteristic has a good symmetry (Fig. 7a). The measured patterns (Fig. 7b-d) show the blocking effect in the range $\vartheta=0^{\circ}-75^{\circ}, \varphi=0^{\circ}-360^{\circ}$. Within this area, there are four significant nulls which are caused by a destructive interference of the diffracted rays around the azimuth positioner. The HW gating technique can slightly improve this negative effect (Fig. 7d). In the standard measurement (Fig. 7b) around the coordinates $\left(\vartheta=160^{\circ}\right.$, $\varphi=110^{\circ}$ ) another null can be seen, which is not expected here. This effect can be slightly reduced using the SW gating technique (Fig. 7c) and it completely vanishes employing the HW gating system (Fig. 7d). Though the amplitude is not compared to the simulated pattern in the range $\vartheta=160^{\circ}-180^{\circ}, \varphi=0^{\circ}-360^{\circ}$, it can be seen that the simulated null only occurs in the measurement acquired with the HW gating technique.

The Fig. 7e-g shows the magnitude of the deviation of the measured patterns from the simulated one. Regarding the chosen area for the evaluation, the average absolute deviation is $2.38 \mathrm{~dB}$ for the standard measurement, $2.07 \mathrm{~dB}$ for the $\mathrm{SW}$ gating, and $1.22 \mathrm{~dB}$ for the $\mathrm{HW}$ gating technique (Table 1).

In Table 1 the results of all investigated antennas are summarized. It can be observed that the HW gating can improve all far-field patterns regarding the amplitude as well as the phase.

\section{Conclusions}

A hardgating system for spherical near-field antenna measurements has been described operating in the time domain. An application specific pulse generator and a sampling receiver enable the identification and the removal of multipath components from the received signal. Compared to conventional SW gating techniques the HW gating principle does not suffer from potential non-physical impulse responses. The presented system can also be employed in only shielded chambers not equipped with absorbing material meeting the requirements for a proper LOS signal detection. The acquired patterns of four different types of antennas show a significant improvement using the HW gating system. The total costs of the instrumentation sum up to only $5 \%$ of standard frequency domain equipment.

Acknowledgements. The authors thank Georg Anastasiadis, Günther Klein, and Oliver Spaccini for the fabrication of the antenna prototypes. Special thanks also to Analog Devices Inc., ON Semiconductor, and Hittite Corp. for the provision of samples of their integrated circuits.

\section{References}

Alenkowicz, H. and Levitas, B.: Using of Sampling Scope for Antenna Measurements in Time and Frequency Domain, in: Proc. 13th International Conference on Microwaves, Radar and Wireless Communications, Wroclaw, Poland, pp. 313-316, May 2000.

Analog Devices Inc.: AD9461, 16-Bit, 130 MSPS IF Sampling ADC, datasheet, http://www.analog.com, Norwood, MA, USA, 2006.

American National Standards Institute/The Institute of Electrical and Electronics Engineers, IEEE Standard Test Procedures for Antennas, ANSI/IEEE Std, 149-1979, 1979.

Autar, K. K.: Lagrangian Interpolation, website, http://www. numericalmethods.eng.usf.edu, February 2003.

Belyaev, B. A., Leksikov, A. A., and Serzhantov, A. M.: Broadband Microstrip Filter, in: Proc. 10th International Crimean Conference on Microwave \& Telecommunicatian Technology, Sevastopol, Ukraine, pp. 365-366, September 2000.

Cox, M. G., Harris, P. M., and Humphreys, D. A.: An algorithm for the Removal of Noise and Jitter in Signals and its Application to Picosecond Electrical Measurement, Numerical Algorithms, 5(10), 491-508, 1993.

Dadic, M. and Zentner, R.: A Technique for Elimination of Reflected Rays from Antenna Measurements Performed in Echoic Environment, Int. J. Electr. Communications, 61(2), 90-94, 2007.

De Jough, R. V., Hajian, M., and Ligthart, L. P.: Antenna TimeDomain Measurement Techniques, IEEE Antennas and Propagation Magazine, 39(5), 7-11, 1997.

Fourestie, B., Altman, Z., Wiart, J., and Azoulay, A.: On the Use of the Matrix-Pencil Method to Correlate Measurements at Different Test Sites, IEEE Transactions on Antennas and Propagation, 47(10), 1569-1573, 1999. 
Fourestie, B. and Altman, Z.: Gabor Schemes for Analyzing Antenna Measurements, IEEE Transactions on Antennas and Propagation, 49(9), 1245-1253, 2001.

Gans, W. L.: The Measurement and Deconvolution of Time Jitter in Equivalent-Time Waveform Samplers, IEEE Transactions on Instrumentation and Measurement, 43(5), 726-732, 1983.

Hansen, J. E.: Spherical Near-Field Antenna Measurements, London, Great Britain:Institute of Electrical Engineers, 1988.

Hartmann, J. and Fasold, D.: A Flexible Hardgating System as a Diagnostic Tool in Single and Double Reflector Compact Ranges, in: Proc. AMTA 1998, Montreal, Canada, pp. 331-336, October 1998.

Henderson, A., James, J. R., Newham, P., and Morris, G.: Analysis of Gating Errors in Time Domain Antenna Measurements, IEE Proceedings on Microwaves, Antennas and Propagation, 136(4), 311-320, 1989.

Higgins, J. R.: A Sampling Theorem for Irregularly Spaced Sample Points, IEEE Transactions on Information Theory, 22(5), 621$622,1976$.

Hittite Microwave Corp.: HMC547LP3, GaAs MMIC SPDT NonReflective Switch, DC - $20 \mathrm{GHz}$, datasheet, http://www.hittite. com, Chelmsford, MA, USA, 2007a.

Hittite Microwave Corp.: HMC565LP5, GaAs PHEMT MMIC Modulator Driver Amplifier, DC - $20 \mathrm{GHz}$, datasheet, http:// www.hittite.com, Chelmsford, MA, 2007b.

Inphi Corp.: 1321TH, $13 \mathrm{GHz}$ Bandwidth 2GS/s THA, datasheet, http://www.inphi-corp.com, Westlake Village, CA, USA, 2006.

Kahrs, M.: 50 Years of RF and Microwave Sampling, IEEE Transactions on Microwave Theory and Techniques, 51(6), 17871805, 2003.

Klembowski, W., Levitas, B., Ponomarev, D., and Proshin, V.: Method of Time Domain Antenna Measurements and it's Application to Field Test of Radar Antenna Subsystem, in: Proc. CIE International Conference of Radar, Beijing, China, pp. 511-514, October 1996.

Leibfritz, M. M., Blech, M. D., Landstorfer, F. M., and Eibert, T. F.: A comparison of software- and hardware-gating techniques applied to near-field antenna measurements, Adv. Radio Sci., 5, 43-48, 2007,

http://www.adv-radio-sci.net/5/43/2007/.
Lestari, A. A., Suksmono, A. B., Kumiawan, A., Bharata, E., Yarovoy, A. G., and Ligthart, L. P.: A Facility for UWB Antenna Measurements in Time Domain, in: Proc. IEEE International Workshop on Antenna Technology: Small Antennas and Novel Metamaterials, Singapore, Singapore, pp. 109-112, March 2005.

Levitas, B. N.: Time Domain Antenna Measurement Systems, in: Proc. The Third International Conference on Ultrawideband and Ultrashort Impulse Signals, Sevastopol, Ukraine, pp. 90-95, September 2006.

Levitas, B. N. and Ponomarev, D. M.: Antenna Measurements in Time Domain, in Proc. IEEE Antennas and Propagation Society International Symposium, Baltimore, MD, USA, 1, 573-576, July 1996.

Loredo, S., Pino, M. R., Las-Heras, F., and Sarkar, T. K.: Echo Identification and Cancellation Techniques for Antenna Measurement in Non-Anechoic Test Sites, IEEE Antennas and Propagation Magazine, 46(1), 100-107, 2004.

ON Semiconductor: MC100EP195 3.3V ECL Programmable Delay Chip, datasheet, http://www.onsemi.com, Phoenix, AZ, USA, December 2006

Paulter, N. G. and Larson, D. R.: The "Median" Method for the Reduction of Noise and Trigger Jitter on Waveform Data, J. Res. National Institute of Standards and Technology, 110(5), 511527, 2005.

Schantz, H. G., Beeler, W., and Dickson, D.: UWB Planar Bulbous Dipole Antennas, in: Proc. IEEE Antennas and Propagation Society International Symposium, Washington, D.C., 2A, 475-478, 2005.

Singh, P. K., Basu, S., and Wang, Y.-H.: Planar Ultra-Wideband Bandpass Filter Using Edge Coupled Microstrip Lines and Stepped Impedance Open Stub, IEEE Microwave and Wireless Components Lett., 17(9), 649-651, 2007.

Souders, T. M., Flach, D. R., and Hagwood, C.: The Effects of Timing Jitter in Sampling Systems, IEEE Transactions on Instrumentation and Measurement, 39(1), 80-85, 1990.

Verspecht, J.: Compensation of Timing Jitter-Induced Distortion of Sampled Waveforms, IEEE Transactions on Instrumentation and Measurement, 32(1), 126-133, 1994. 